\title{
Vitreolensectomy in Marfan's syndrome
}

\begin{abstract}
Purpose To evaluate the effectiveness of pars plana vitreolensectomy in the management of subluxed lenses associated with Marfan's syndrome and to assess the need for intraoperative retinal photocoagulation to prevent post-operative retinal detachment. Method A retrospective review was carried out of 40 eyes of patients with Marfan's syndrome who underwent pars plana vitreolensectomy for subluxed lenses.

Results All patients demonstrated stable or improved visual acuity following surgery with a low incidence of complications.

Conclusions Pars plana vitreolensectomy is a safe and effective treatment for subluxed lenses in patients with Marfan's syndrome. It appears that intraoperative prophylactic laser treatment need only be applied to areas of lattice degeneration to limit the incidence of post-operative retinal detachment.
\end{abstract}

Key words Lattice degeneration, Marfan's syndrome, Retinal detachment, Subluxed lens, Vitreolensectomy

Marfan's syndrome is the most frequent cause of heritable lens subluxation. Between $50 \%$ and $80 \%$ of Marfan's syndrome patients have some degree of lens subluxation, which is usually bilateral and symmetrical. Although it characteristically occurs in a superotemporal direction the subluxation may occur in any meridian, or the lens may dislocate into the vitreous cavity or anterior chamber. In the majority of cases lens subluxation results in optical defocus and distortion with reduction in

A.D. Hubbard

D.G. Charteris

R.J. Cooling

Moorfields Eye Hospital

London EC IV 2PD, UK

A.D. Hubbard las

Roy Harfitt Eye Unit

Sutton Hospital

Cotswold Road

Sutton

Surrey, UK

Tel: +44 (0)1812962000

The authors have no proprietary interest in any instrument or technique described in this article visual acuity. If the ametropia cannot be corrected satisfactorily with spectacles or contact lenses then lens removal may be indicated. Other ocular features of Marfan's syndrome include high axial myopia, poor pupillary dilation, peripheral iris transillumination and glaucoma. Retinal detachment is a common complication of Marfan's syndrome and is related to a combination of high axial myopia, lens subluxation and aphakia. Phakic eyes of normal axial length with non-subluxed lenses are not associated with a significantly increased risk of retinal detachment. ${ }^{1}$
A.D. HUBBARD, D.G. CHARTERIS,

R.J. COOLING
The surgical management of subluxed lenses in patients with Marfan's syndrome by intracapsular or extracapsular lens extraction techniques has previously been associated with a high incidence of intraoperative and postoperative complications, especially vitreous loss and retinal detachment. ${ }^{2,3}$ The previously reported high incidence of post-operative retinal detachment is believed to be related to the predisposition of these eyes to retinal detachment combined with surgical techniques that result in vitreous incarceration and traction. $^{1-\gamma}$ More recent studies have claimed reduced complication rates when subluxed lenses are removed via closed intraocular microsurgical techniques, but the number of patients with Marfan's syndrome in these studies has been small and their outcome has not been studied independently from that of patients with subluxed lenses from other causes. $^{4-\tau}$ The outcome of pars plana vitreolensectomy in Marfan's syndrome deserves individual attention due to the perceived increased risk of lens removal, by any technique, and the propensity to retinal detachment in Marfan's patients.

The aim of this study was to assess the indications for, and surgical outcome of, pars plana vitreolensectomy for lens subluxation in Marfan's syndrome and to evaluate the need for intraoperative retinal laser or cryotherapy in the prevention of post-operative retinal detachment.

\section{Patients and methods}

Forty eyes of 30 patients with Marfan's syndrome who had undergone pars plana vitreolensectomy were retrospectively reviewed. Twenty patients were male and 10 female with a mean age of 33 years (range 6-67 years). The mean period of follow-up was 18 months (range 6-124 months).

All patients underwent three-port pars plana vitreolensectomy under general anaesthesia. All patients had a complete vitrectomy with removal of the posterior hyaloid face. Lensectomy was performed by a combination of ultrasonic fragmentation and cutting/ aspiration. Selected patients also underwent laser or cryotherapy to areas of lattice degeneration, simultaneous retinal detachment 
repair and insertion of a phacoprosthesis at the time of primary surgery. In the retinal detachment group, all patients had lensectomy in association with vitrectomy, internal retinal detachment repair and intraocular tamponade to aid intraoperative visualisation, reduce the risk of post-operative retinal detachment and prevent the need for future lens surgery. In the absence of an associated retinal detachment prophylactic laser or cryotherapy was applied to lattice degeneration only (9 patients). Three hundred and sixty degree prophylactic treatment was not performed. Only two eyes received cryotherapy to the retina posterior to the entry sites.

The main outcome measures were: indications for surgery, visual recovery, retinal surgery/prophylaxis and complications.

\section{Results}

The clinical indications for surgery are presented in Table 1. The most common reason for intervention was poor vision due to rapidly changing or uncorrectable refractive error caused by lens subluxation.

The visual outcome is summarised in Fig. 1. No patients in our series suffered a reduction in visual acuity as a result of surgical intervention, with a mean improvement of 2.8 lines Snellen acuity.

Thirty-seven eyes were aphakic post-operatively and corrected with contact lenses in all cases. Three eyes had sutured posterior chamber intraocular lens implants (two as a primary procedure and one as a secondary procedure).

\section{Retinal detachment}

Seven eyes had a coexisting retinal detachment at the time of primary surgery (mean age 31 years). Six eyes had retinal detachments with a subluxed lens (one gave a history of minor trauma). One eye had a lens that had previously been noted to be subluxed but had become dislocated into the vitreous cavity following blunt trauma which also precipitated a retinal detachment. One eye had undergone a successful external retinal detachment repair 11 years previously and had developed recurrent retinal detachment. In addition to vitreolensectomy all eyes underwent scleral buckling (six were encircled and one had a local circumferential explant), laser/cryotherapy to retinal tears and areas of lattice degeneration and internal tamponade with $\mathrm{SF}_{6}$ or $\mathrm{C}_{3} \mathrm{~F}_{8}$ gas at the primary procedure.

Table 1. Indications for surgery

\begin{tabular}{lc}
\hline Subluxed lens with reduced visual acuity & 26 \\
$\quad$ (uncorrectable refractive error) & 7 \\
Subluxed lens and retinal detachment & 2 \\
Subluxed cataractous lens & 4 \\
Phacolytic glaucoma & 1 \\
Phacomorphic glaucoma & 40 \\
Total & \\
\hline
\end{tabular}

Four eyes had an attached retina post-operatively and required no further intervention (mean follow-up 16.5 months). One eye (HM pre-operatively; 6/18 final visual acuity) with a sub-total retinal detachment preoperatively had a persistent inferior retinal detachment post-operatively and a second vitreoretinal procedure (internal search/membranectomy/silicone oil) was undertaken 2 weeks after primary surgery. This patient developed a small persistent inferior tractional retinal detachment that was not subject to further surgery and remained stable during the 2 years of available followup. The results of surgery in the remaining two eyes were initially successful but recurrent retinal detachment associated with proliferative vitreoretinopathy developed 3 months post-operatively in both patients. Each of these cases achieved satisfactory results following a single further vitreoretinal procedure (Fig. 1).

Two eyes $(6 \%)$ that underwent vitreolensectomy in the absence of pre-operative retinal detachment developed retinal detachment post-operatively. One was evident immediately post-operatively and resulted from an entry site break and the other developed 6 months post-operatively from a retinal break in an area of untreated lattice degeneration. Both retinas were reattached successfully following further surgery (Fig. 1).

Three eyes in our series had undergone external retinal detachment repair prior to vitreolensectomy. Two of these patients gave a history of preceding minor trauma. None of these retinas redetached after vitreolensectomy.

Refractive state was available in only two of the eyes that presented with retinal detachment. These eyes were both high axial myopes with refractive errors of $-22 \mathrm{D}$ and $-26.5 \mathrm{D}$ (spherical equivalent).

\section{Retinal prophylaxis}

Eleven eyes had lattice retinal degeneration noted in the operated eye. In two cases this was noted in association with a retinal detachment, but it was unclear from the patient records whether the retinal breaks responsible for the detachment arose in lattice. Nine of the 11 eyes had the lattice degeneration treated intraoperatively with laser or cryotherapy (6 laser, 3 cryotherapy).

Of the seven eyes that received laser/cryotherapy to lattice degeneration in the absence of retinal detachment, none developed retinal detachment in the follow-up period (mean 11 months).

Two eyes had lattice degeneration noted preoperatively but received no laser/cryotherapy. One of these eyes developed a retinal detachment secondary to a retinal tear in an area of lattice degeneration 6 months post-operatively (which was subsequently successfully repaired), whilst the other eye has maintained an attached retina for 15 months post-operatively.

Lattice degeneration was noted in six fellow eyes either pre- or intra-operatively. Four of these eyes received laser/cryotherapy at the time of primary surgery whilst the others remained untreated. None of 


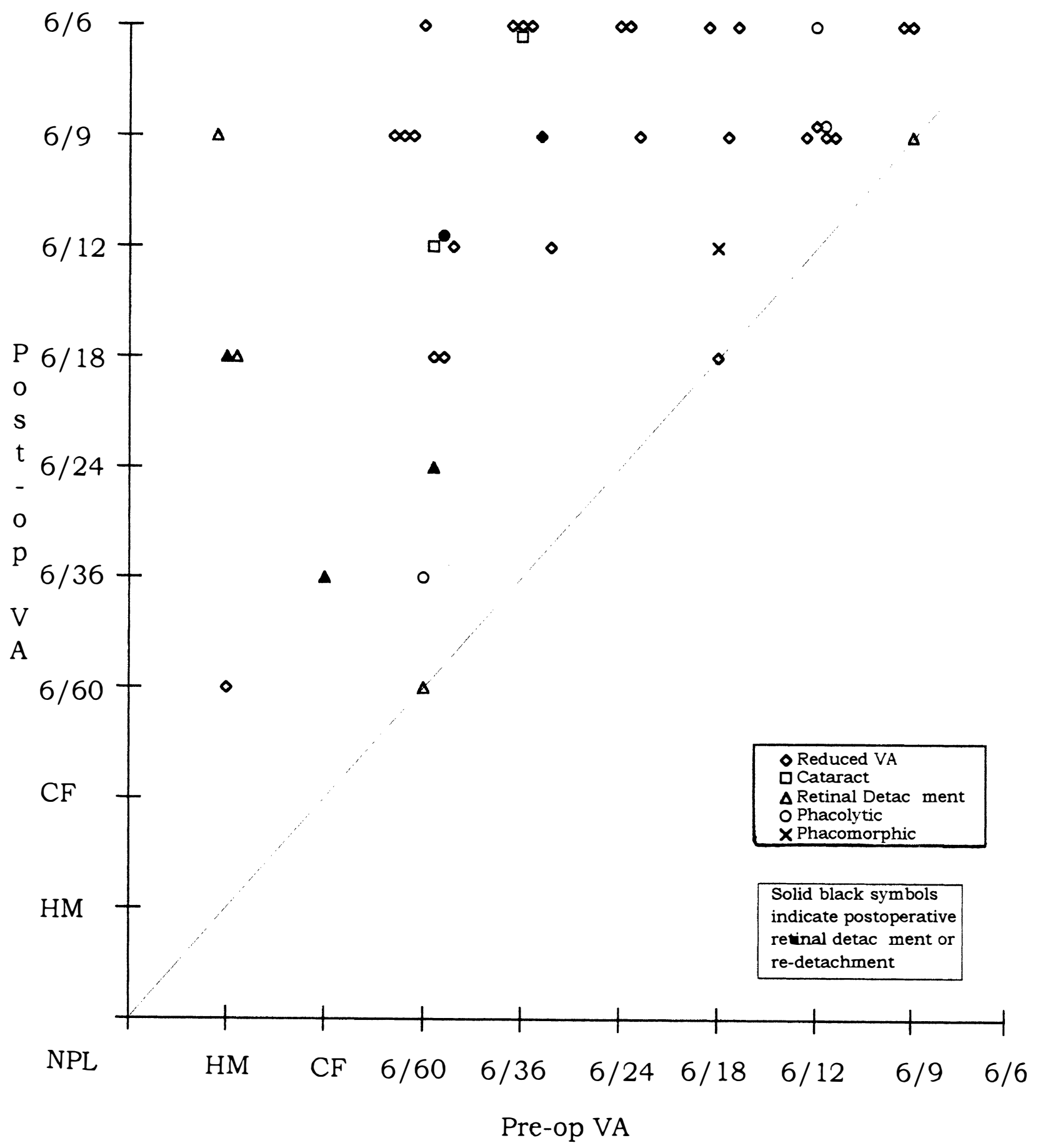

Fig. 1. Visual outcome.

the fellow eyes developed retinal detachment in the follow-up period (mean 14 months).

None of the patients in our series underwent $360^{\circ}$ prophylaxis either pre- or intra-operatively.

\section{Glaucoma}

Four eyes with phacolytic glaucoma were present in our series (mean age 53 years). All had cataractous lenses that had dislocated into the vitreous cavity, usually long before presentation. Glaucoma resolved in all cases following primary surgery without the need for glaucoma filtration surgery or topical anti-glaucoma medication.
One eye presented with phacomorphic glaucoma caused by the lens dislocating into the anterior chamber (25-year-old patient). The presenting attack was broken by pupillary dilation, allowing the lens to drop back behind the pupillary plane, and subsequent pupillary constriction. Vitreolensectomy was performed electively and without complication.

\section{Discussion}

Subluxation of the crystalline lens may cause visual symptoms that vary in severity depending on the degree of lens displacement. The lens may remain in its normal position, subluxing slightly backwards, with minimal 
refractive error. Lenticular myopia may result from increased curvature of the lens because of zonular release. A displaced lens may be tilted causing myopia and astigmatism that may be impossible to correct optically. If the lens is sufficiently subluxed an aphakic correction will be necessary and visual disturbance may be minimal. A subluxed lens may also cause phacomorphic or phacolytic glaucoma and may contribute to vitreous changes leading to the development of retinal detachment. Although there is little dispute that lens removal is indicated in these circumstances, there has traditionally been a reluctance to operate on eyes with a moderate reduction in acuity from optical distortion due to the reported high risk of complications and poor visual outcome.

Jarrett $^{2}$ in 1967 and Cross and Jensen ${ }^{3}$ in 1973 reported the results of removal of subluxed lenses in Marfan's patients via a corneal section using a variety of surgical techniques (discission, aspiration, intra- and extracapsular extraction). They reported an improvement in visual acuity in approximately $60 \%$, a vitreous loss rate of $25-30 \%$, and a post-operative retinal detachment rate of $20-30 \%$ in their combined series of 73 patients.

In 1979, Peyman et al. ${ }^{4}$ reported successful extraction of subluxed lenses via the pars plana route. Similar successes have been reported more recently, ${ }^{5-8}$ but the numbers of Marfan's syndrome patients in these series were small and their outcome was not analysed independently from other causes of lens subluxation

Our series has demonstrated that good visual results can be achieved with pars plana vitreolensectomy for subluxed lenses in Marfan's syndrome with an acceptable complication rate. The post-operative incidence of retinal detachment was $6 \%$. This improved retinal detachment rate can be attributed to reduced tractional forces on the retina as a result of the vitrectomy, intraoperative treatment of predisposing retinal pathology and the avoidance of vitreous incarceration in a corneal section.

In all our cases retinal prophylaxis was applied to areas of lattice retinal degeneration where this was felt to be indicated by the operating surgeon. It is interesting to note that one of the two post-operative retinal detachments in our series arose in an area of lattice retinal degeneration which was not treated at the time of primary surgery. It has been suggested that selected patients with known tendency to retinal detachment undergoing vitrectomy should have routine $360^{\circ}$ retinal prophylaxis applied at the primary procedure. ${ }^{9}$ Our series suggests that in Marfan's syndrome a more limited application of laser/cryotherapy only to areas of lattice degeneration is adequate prophylaxis.

In patients presenting with a retinal detachment associated with a subluxed lens our series shows that both conditions may be treated successfully at a single surgical procedure with good visual results. In some cases the retinal detachment is amenable to conventional scleral buckling surgery and this may be performed with the subluxed lens in situ, which may then be removed at a later date if necessary. However, removal of subluxed lens and retinal detachment repair performed together as a primary procedure has several advantages; the subluxed lens and associated vitreous changes are often causally related to the retinal detachment, removal of subluxed lens allows better intraoperative retinal visualisation, visual recovery is often more rapid following removal of a subluxed or cataractous lens, and surgical time and patient inconvenience are kept to a minimum.

In our series patients presenting with phacolytic or phacomorphic glaucoma responded well to vitreolensectomy with no need for further antiglaucomatous measures. We would therefore advise early intervention in all such cases.

Following vitreolensectomy the majority of our patients were rendered aphakic and refractive error subsequently corrected with contact lenses. As a group refractive correction with contact lenses appeared to be extremely well tolerated, probably because many of the patients had been contact lens wearers before surgery. Three patients had sutured posterior chamber intraocular lens implants (two with the primary procedure and one as a secondary procedure). We would suggest that this is a satisfactory way of correcting refractive error if the patient is likely to be contact lens intolerant. The increased incidence of glaucoma in Marfan's syndrome, often associated with angle abnormalities, is a relative contraindication to the use of anterior chamber intraocular lens implants, although a recent paper has reported their use in a small number of Marfan's syndrome patients to correct aphakia following pars plana vitreolensectomy without reported adverse sequelae. $^{10}$

The youngest patient undergoing pars plana vitreolensectomy in our series was 6 years old. When considering surgery in young children it should be remembered that the pars plana does not reach adult proportions until the age of 7 years. Hakin et al. ${ }^{7}$ and Plager et al. ${ }^{8}$ have reported good results using a two-port limbal lensectomy and anterior vitrectomy in the paediatric age group. Limbal lensectomy requires less instrumentation and the avoidance of a posterior infusion line reduces possible trauma to the vitreous base and retina. This approach may be more appropriate for the non-specialist vitreoretinal surgeon but it is associated with incomplete removal of the vitreous, and vitreous incarceration and traction may occur which is associated with an increased post-operative retinal detachment rate in Marfan's syndrome patients. ${ }^{2,3}$ In these circumstances the case for $360^{\circ}$ retinal prophylaxis is more compelling. The pars plana approach has advantages over the limbal approach in that retinal pathology may be treated directly and vitreous removal can be more complete with reduced tractional forces. In older patients with a hard lens requiring ultrasonic fragmentation before aspiration, the pars plana approach has definite advantages as it allows easy access to the vitreous cavity should posterior displacement of lens matter occur. 
In summary, pars plana vitreolensectomy combined with retinal prophylaxis to areas of lattice retinal degeneration is a safe and effective surgical technique for lens subluxation in patients with Marfan's syndrome.

\section{References}

1. Nelson LB, Maumenee IH. Ectopia lentis. Surv Ophthalmol 1982;27:143-60.

2. Jarrett WH. Dislocation of the lens: a study of 166 hospitalized cases. Arch Ophthalmol 1967;78:289-96.

3. Cross HE, Jensen AD. Ocular manifestations in the Marfan syndrome and homocystinuria. Am J Ophthalmol 1973;75:405-20.
4. Peyman GA, et al. Management of subluxed and dislocated lenses with the vitreophage. Br J Ophthalmol 1979;63:771-8.

5. Reese PD, Weingeist TA. Pars plana management of ectopia lentis in children. Arch Ophthalmol 1987;105:1202-4.

6. Girard LJ, et al. Subluxed (ectopic) lenses in adults. Ophthalmology 1990;97:462-5.

7. Hakin $\mathrm{KN}$, et al. Management of the subluxed crystalline lens. Ophthalmology 1992;99:542-5.

8. Plager DA, et al. Surgical treatment of subluxed lenses in children. Ophthalmology 1992;99:1018-21.

9. McLeod D. Giant retinal tears after central vitrectomy. Br J Ophthalmol 1985;69:96-8.

10. Koenig SB, Mieler WF. Management of ectopia lentis in a family with Marfan syndrome. Arch Ophthalmol 1996;114:1058-61. 